\title{
Induction of Rare Conformation of Oligosaccharide by Binding to Calcium-dependent Bacterial Lectin: X-ray Crystallography and Modelling Study
}

Martin Lepsik, ${ }^{1 *}$ Roman Sommer, ${ }^{2,3,4}$ Sakonwan Kuhaudomlarp, ${ }^{1}$ Mickaël Lelimousin, ${ }^{1}$ Emanuele Paci, ${ }^{5}$ Annabelle Varrot, ${ }^{1}$ Alexander Titz ${ }^{2,3,4}$ and Anne Imberty ${ }^{1 *}$

${ }^{1}$ Univ. Grenoble Alpes, CNRS, CERMAV, 38000 Grenoble, France

2 Chemical Biology of Carbohydrates, Helmholtz Institute for Pharmaceutical Research Saarland (HIPS), Helmholtz Centre for Infection Research, D-66123 Saarbrücken, Germany

${ }^{3}$ Deutsches Zentrum für Infektionsforschung (DZIF), Standort Hannover-Braunschweig, Germany

${ }^{4}$ Department of Pharmacy, Saarland University, D-66123 Saarbrücken, Germany

${ }^{5}$ Astbury Centre \& School of Molecular and Cellular Biology, University of Leeds, Leeds, U.K.

* Corresponding author. Martin Lepsik (martin.lepsik@cermav.cnrs.fr) \& Anne Imberty (anne.imberty@ cermav.cnrs.fr)

\section{KEYWORDS}

Lectin, carbohydrate; calcium ion; Quantum effects; Molecular Dynamics; $N$-Acetyl 


\begin{abstract}
Pathogenic micro-organisms utilize protein receptors in adhesion to host tissues, a process that in some cases relies on the interaction between lectin and human glycoconjugates. Oligosaccharide epitopes are recognized through their three-dimensional structure and their flexibility is a key issue in specificity. In this paper, we analyse by X-ray crystallography the structures of the lectin LecB from two strains of Pseudomonas aeruginosa in complex with Lewis x oligosaccharide present on cell surfaces of human tissues. An unusual conformation of the glycan was observed in all binding sites with a non-canonical syn orientation of the $\mathrm{N}$ acetyl group of $\mathrm{N}$-acetyl-glucosamine. A PDB-wide search revealed that such an orientation occurs only in $2 \%$ of protein/carbohydrate complexes. Theoretical chemistry calculations showed that the observed conformation is unstable in solution but stabilised by the lectin. A reliable description of LecB/Lewis x complex by force field-based methods had proven as especially challenging due to the special feature of the binding site, two closely apposed $\mathrm{Ca}^{2+}$ ions which induce strong charge delocalisation. By comparing various force-field parametrisations, we design general protocols which will be useful in near future for designing carbohydrate-based ligands (glycodrugs) against other calcium-dependent protein receptors.
\end{abstract}




\section{INTRODUCTION}

Pseudomonas aeruginosa is a gram-negative bacterium, which acts as an opportunistic pathogen responsible for severe bronchopulmonary infections notably in cystic fibrosis patients. $P$. aeruginosa disposes of many soluble virulence factors, including two lectins, LecA (PA-IL) and LecB (PA-IIL), with specificity for galactose (Gal) and fucose (Fuc), respectively [1, 2]. Structurally, LecB is a homotetramer with four binding sites, each of which contains two $\mathrm{Ca}^{2+}$ atoms [3] that mediate the binding of Fuc accompanied by a strong charge delocalisation [4]. The high affinity to Fuc results in strong attachment to branched fucosylated oligosaccharides (Fig. 1A and 1B) present in the human blood group epitopes such as the $\mathrm{AB}(\mathrm{H})$ and Lewis series (Fig. 1C) [5].

A

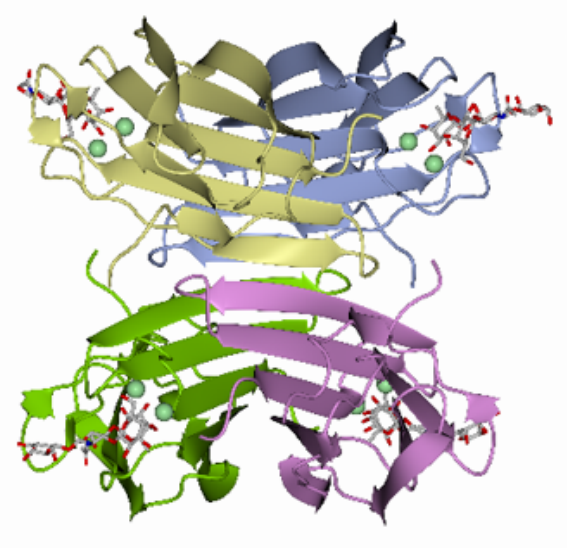

C
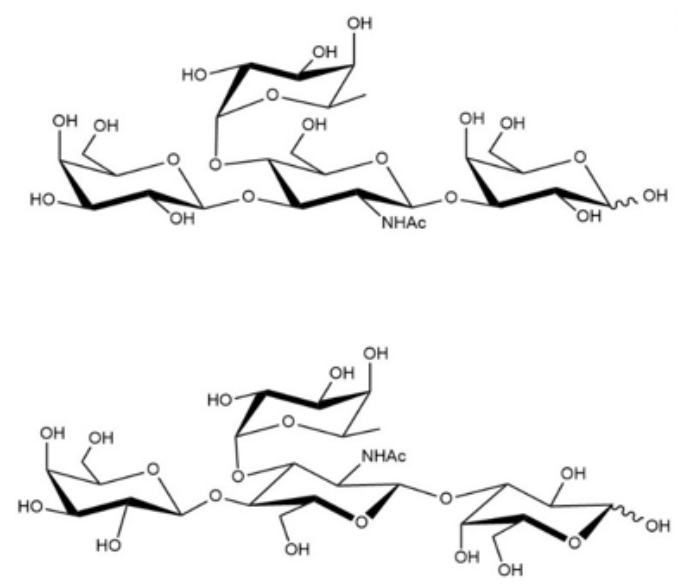

B

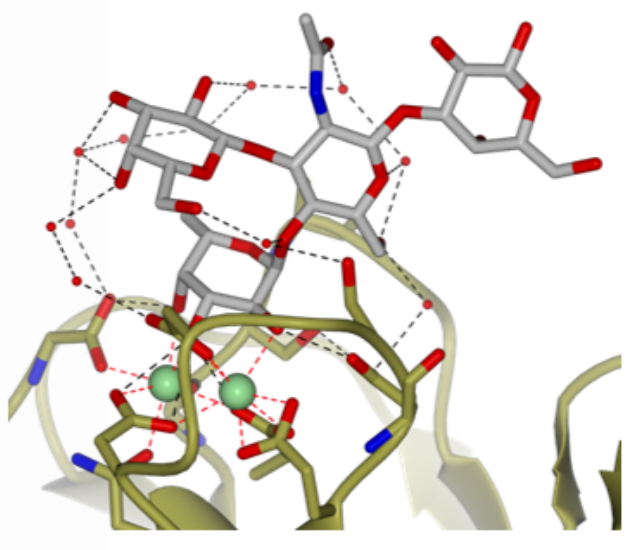

D

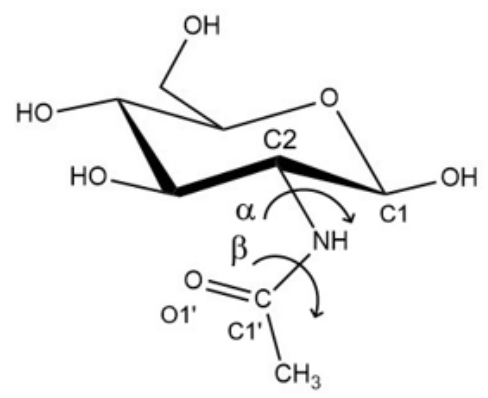

Figure 1. Structure of LecB and fucosylated ligands. A Overall view of LecB $\mathrm{BA}_{\mathrm{P} 14}$ tetramer (coloured cartoon) with two calcium ions (green spheres) and one Le $\mathrm{C}^{\mathrm{a}}$ tetrasaccharide bound (sticks) in each binding site (PDB 5A6Z)[6]. B Zoom in the binding site displaying the orientation of the $N$-acetyl group in the solvent. C The chemical structures of $\mathrm{Le}^{\mathrm{a}}$ (top) and 
Le $^{\mathrm{x}}$ (bottom) tetrasaccharides. D The chemical structure of D-GlcNAc with definitions of $\alpha$ and $\beta$-dihedrals [7].

Among human epitopes, LecB demonstrated higher affinity for Lewis a oligosaccharide (Le ${ }^{\mathrm{a}}$ : Fuc $\alpha(1-4)$ [Gal $\beta(1-3)]$ GlcNAc) which was rationalised by X-ray structure of the complex [8]. In the binding site, Fuc coordinates the two calcium ions via its hydroxyl groups, which moreover also act as hydrogen bond donors to acidic amino acids of the binding site. Furthermore, $N$-acetyl-glucosamine (GlcNAc) creates an additional hydrogen bond with the protein surface. Different $P$. aeruginosa strains have been investigated [6, 9], since the classical PAO1 laboratory strain is not the only to be involved in clinical infection. LecB from strains PA14 ( LecB $\left._{\mathrm{PA} 14}\right)$ and PAO1 ( LecB $\left._{\mathrm{PAO} 1}\right)$ differ by $13 \%$ in sequences, but both bind to $\mathrm{Le}^{\mathrm{a}}$ with high affinity as determined by titration microcalorimetry $\left(K_{\mathrm{d}}\right.$ of $78 \mathrm{nM}$ and $170 \mathrm{nM}$, respectively) [6]. Binding to Lewis x epitope (Le ${ }^{\mathrm{x}}$ : Fuc $\alpha(1-3)$ [Gal $\left.\beta(1-4)\right]$ GlcNAc) is almost as efficient as to $\mathrm{Le}^{\mathrm{a}}$ with affinities to LecB $\mathrm{PA} 14_{4}$ and $\mathrm{LecB}_{\mathrm{PAO} 1}$ of 90 and $400 \mathrm{nM}$, respectively) $[6,8]$. This was unexpected since the orientation of GlcNAc in Le $^{\mathrm{x}}$ is different from that in $\mathrm{Le}^{\mathrm{a}}$ and the $\mathrm{N}$-acetyl was predicted to create steric conflict with the protein surface [3].

The Lewis epitopes are the targets for viral receptors, such as those of noroviruses, and for a number of bacterial lectins $[5,10]$. These oligosaccharides are usually described as rigid due to their branched structure, that results in stacking between Fuc and Gal rings, steric hindrance of the $N$-acetyl group of $N$-acetylglucosamine (GlcNAc) and the presence of an unconventional $\mathrm{CH}^{\cdots} \mathrm{O}$ hydrogen bond [11-13]. The conformation in solution of Lewis oligosaccharides is referred to as the "closed" conformation, which is the one recognized by most protein receptors. However, a class of lectins from fungi and bacteria has been reported to induce a conformation change in $\mathrm{Le}^{\mathrm{x}}$ and a series of "open" conformations were observed by X-ray crystallography and rationalised by molecular dynamics (MD) calculations [14]. Characterising the interaction of bacterial lectins with their natural ligands is of interest for the development of anti-infective compounds that are able to inhibit the adhesion of bacteria or the formation of biofilm [15]. For example, the structure of LecB complexed with Le ${ }^{\mathrm{a}}$ was used for the design of fucose derived glycomimetics with aromatic aglycone mimicking the GlcNAc ring [16] and mannose/fucose derived glycomimetics carrying sulphonamide substituents $[17,18]$.

We describe here the crystal structures of LecB from PAO1 and PA14 strains in complex with Le $^{\mathrm{x}}$ and we analyze the conformation of GlcNAc (Fig. 1D) by quantum mechanical (QM) and molecular dynamics (MD) calculations. The presence of bridging calcium ions and water molecules in the binding site necessitates special care for force-field parameterization as previously demonstrated $[19,20]$ and several approaches are compared. The derived 
method will be of general interest in the future for design of active compounds against calcium-containing lectins from pathogens.

\section{RESULTS}

\section{Crystal structures of LecB complexed with Le ${ }^{x}$}

$\mathrm{LecB}_{\mathrm{PA} 14}$ and $\mathrm{LecB}_{\mathrm{PAO} 1}$ were co-crystallised with the $\mathrm{Le}^{\mathrm{x}}$ tetrasaccharide, resulting in crystals in $\mathrm{P} 2{ }_{1}$ space group diffracting to $1.6 \AA$ and $1.8 \AA$, respectively. The structures were solved by molecular replacement and details are listed in Table S1. Clear electron density was observed for the whole oligosaccharides in all four binding sites of both structures, except for the reducing-end $\mathrm{Gal}$ in LecB $\mathrm{PAO}_{\mathrm{PA}}$ (Fig. S1). Consistent with previous findings, both structures are homotetramers with four binding sites, each of which contains two $\mathrm{Ca}^{2+}$ atoms[3,6]. In the same manner as for complexes with Fuc [3] or $\operatorname{Le}^{\mathrm{a}}[6,8]$, the calcium atoms mediate the binding of Fuc residue in $\mathrm{Le}^{\mathrm{x}}$ via three hydroxyl oxygens (O2, O3 and $\left.\mathrm{O} 4\right)$. Additionally, the three hydroxyl groups of Fuc can form several direct hydrogen bonds with amino acid residues within the binding site (N21:O, D96:OD1, D99:OD2, D101:OD2 and G114*:OXT). The recognition of Fuc residue by $\mathrm{LecB}_{\mathrm{PA} 14}$ and $\mathrm{LecB}_{\mathrm{PAO} 1}$ are in complete agreement with previous observations $[3,6]$.

In order to rationalise the recognition of $\mathrm{Le}^{\mathrm{x}}$ by the $\mathrm{LecB}$ variants ( $\mathrm{LecB}_{\mathrm{PA} 14}$ and $\left.\mathrm{LecB}_{\mathrm{PAO} 1}\right)$ in relation to the amino acid mutations in their binding site (S97/A23 vs G97/S23, respectively), detailed comparison of their complexes with $\mathrm{Le}^{\mathrm{x}}$ was performed. The serine at position 97 in $\mathrm{LecB}_{\mathrm{PA} 14}$ provides a hydrophilic environment and organises waters $\mathrm{W} 1, \mathrm{~W} 2$, and $\mathrm{W} 3$ to form several hydrogen-bond bridges between the protein and the carbohydrate (Fig. 2A). As a result of the $\mathrm{S} 97 \mathrm{G}$ variation in $\mathrm{LecB}_{\mathrm{PAO} 1}$, W2 and $\mathrm{W} 3$ are absent in $\mathrm{LecB}_{\mathrm{PAO} 1}-\mathrm{Le}^{\mathrm{x}}$ complex and the water-coordinated interaction is replaced by a direct hydrogen bond between the $\mathrm{N}$ acetyl of the GlcNAc and the D96 side chain Fig. 2B). The W1 interactions with the carbohydrates are retained in both proteins. From the protein side, W1 is held by an H-bond from the N98:N backbone.

The A23 variant in $\mathrm{LecB}_{\mathrm{PA} 14}$ constitutes (together with $\mathrm{T} 45$ ) a hydrophobic pocket which accepts the Fuc:C6 methyl (Fig. 2C), as described previously for LecB PA14-Le ${ }^{\mathrm{a}}$ [6]. Further toward the bulk solvent, a water network links the protein with the carbohydrate (Fig. 2C). $\mathrm{W} 4$, present in this water network forms a direct contact with the non-reducing Gal (Fig. 2A, $\mathrm{C})$. In LecB $\mathrm{PAO}_{1}, \mathrm{~S} 23$ was found in two alternate conformations, one of which provides a direct H-bond to GlcNAc:O1' (Fig. 2D). The presence of the S23 polar side chain seems to disrupt the water network described in LecB $\mathrm{PA} 14_{4}$ complex (Fig. 2D). 
A.
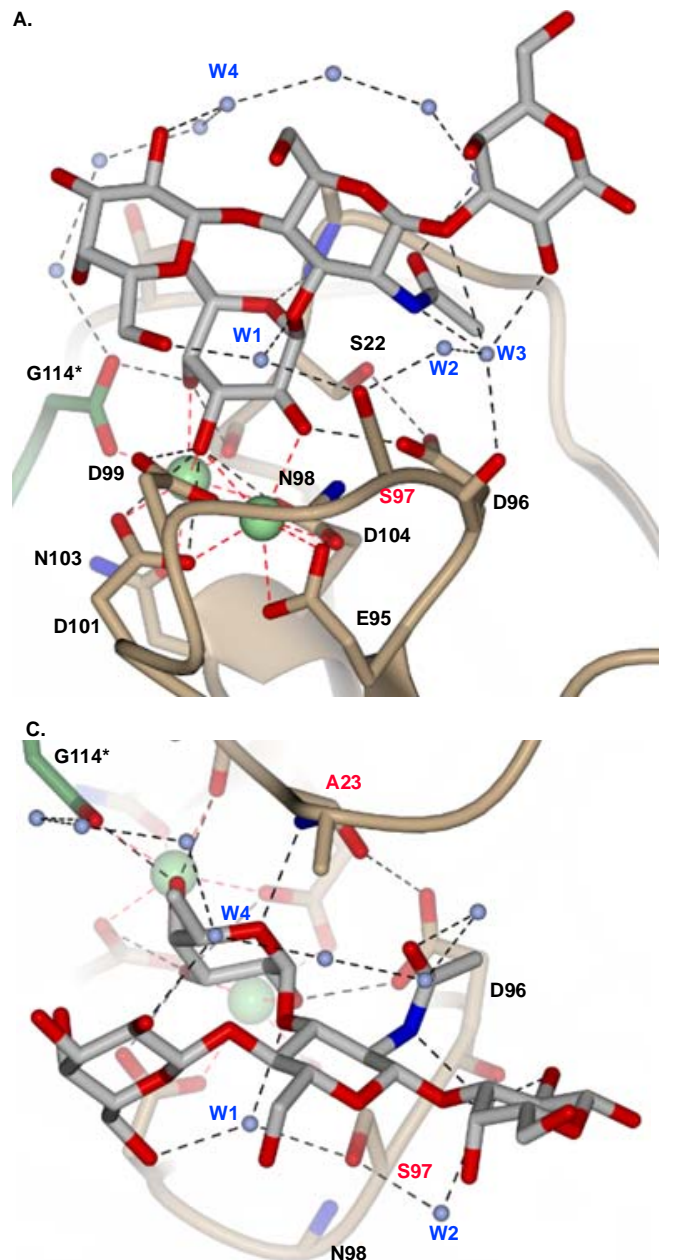

E.

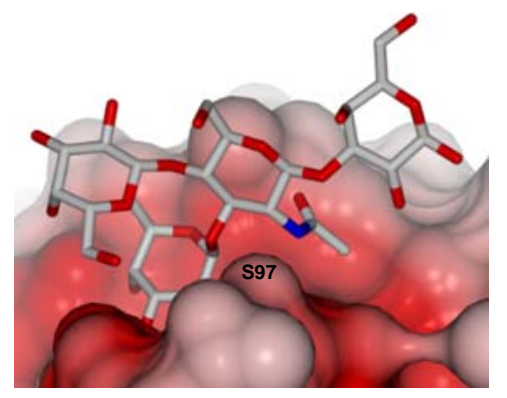

B.

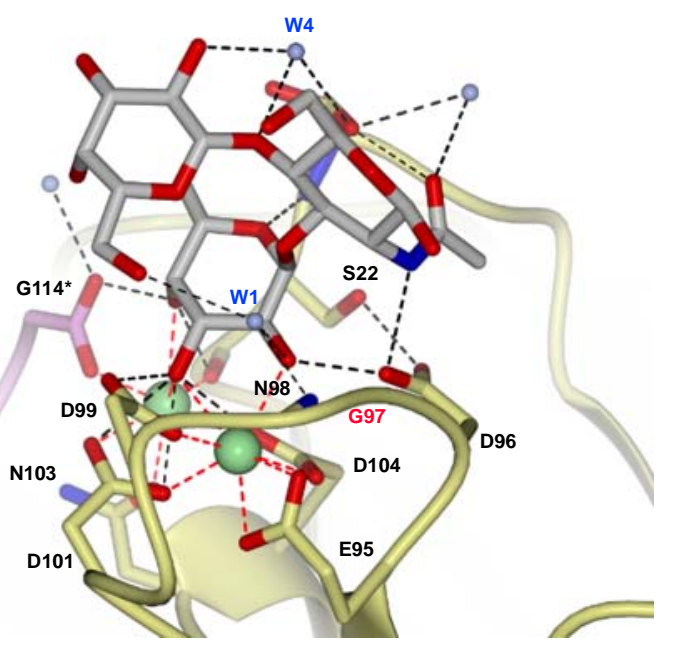

D.

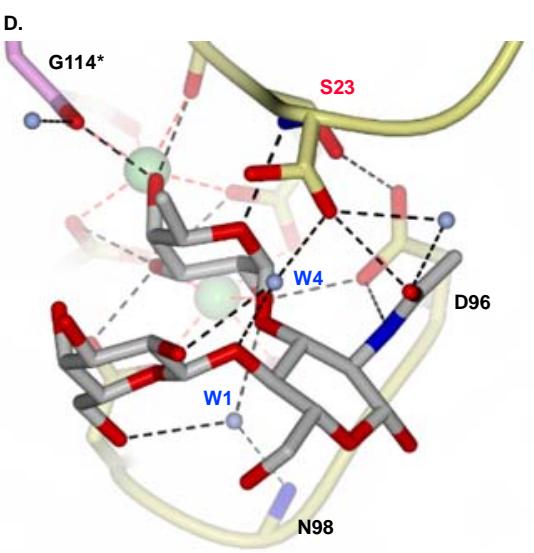

F.

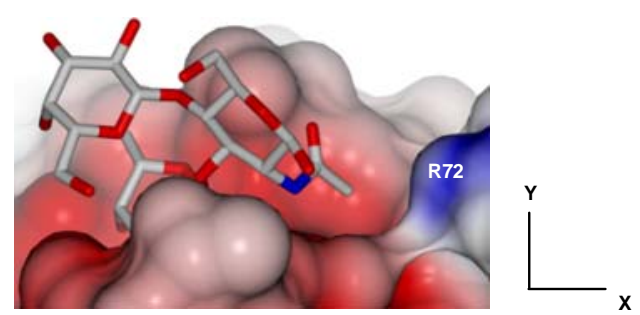

Figure 2. Comparison of crystallographic structures of $\operatorname{Le}^{\mathrm{x}}$ in complex with $\operatorname{LecB}_{\mathrm{PA} 14}(\mathbf{A}, \mathbf{C})$ and $\mathrm{LecB}_{\mathrm{PAO} 1}(\mathbf{B}, \mathbf{D})$. The views in $\mathbf{C}, \mathbf{D}$ correspond to a $90^{\circ}$ clockwise rotation of $\mathbf{A}, \mathbf{B}$, respectively, around the $\mathrm{x}$ axis. $\mathrm{Ca}^{2+}$ atoms are represented by green sphere. The protein backbones of $\mathrm{LecB}_{\mathrm{PA} 14}$ and $\mathrm{LecB}_{\mathrm{PAO} 1}$ are coloured in light brown and yellow, respectively. G114* is the N-ter residue of the adjacent monomer and is coloured in green (in LecB $\mathrm{PA} 14_{4}$ ) or pink (in $\mathrm{LecB}_{\mathrm{PAO} 1}$ ). Water molecules are shown as ice-blue spheres. $\mathrm{Le}^{\mathrm{x}}$ is coloured in light grey (note that in LecB $\mathrm{PAO}_{1}$, the reducing galactose residue could not be modelled due to poor electron density; Fig. S1). $\mathbf{E}$ and $\mathbf{F}$ are surface representation of $\mathbf{A}$ and $\mathbf{B}$, respectively. The surfaces are coloured by electrostatic potential (red; negative, blue; positive). Note that R72 is present in both LecB variants, but the side chain of R72 was only modelled in LecB $\mathrm{BAO}_{\text {, }}$ 
thus is only visible in $\mathbf{F}$. The amino acid variants are labelled in red. The figure was rendered in CCP4MG.

Due to the amino acid variations, some differences are observed in the interaction between $\mathrm{Le}^{\mathrm{x}}$ and the two LecB variants. While the Fuc binding is virtually unchanged, the $\Phi, \Psi$ torsion angles of the $\alpha$ Fuc1-3GlcNAc glycosidic linkage in chains $\mathrm{A}$ and $\mathrm{C}$ differ by up to $16.5^{\circ}$ and $11.5^{\circ}$, between $\mathrm{LecB}_{\mathrm{PAO} 1}$ and $\mathrm{LecB}_{\mathrm{PA} 14}$ (Table $\mathrm{S} 2$ ), which results in a slight change in the interactions. However, in chain D, these torsions are very similar, with changes of 0.9 and $0.5^{\circ}$ (Table S2). Main observed differences are in the interaction between GlcNAc and the protein surface. In $\mathrm{LecB}_{\mathrm{PAO} 1}$, the $\mathrm{NH}$ of GlcNAc is close enough to the Asp96 side chain to form a direct hydrogen bond (Fig. 2B, D). In $\mathrm{LecB}_{\mathrm{PA} 14}$, the GlcNAc ring is pushed slightly away and instead of a direct hydrogen bond, a water bridge via $\mathrm{W} 3$ is observed between $\mathrm{NH}$ of GlcNAc and the backbone oxygen of Asp96 (Fig. 2A, C). In addition, the S97G mutation in Lec $A_{\mathrm{PAO} 1}$ provides a hydrophobic patch, enabling a closer contact of the GlcNAc ring with the protein surface, which is not observed in $\mathrm{LecB}_{\mathrm{PA} 14}$ due to the polar $\mathrm{OH}$ group of $\mathrm{S} 97$ side chain (Fig. 2E and F).

In the crystal structures of both $\mathrm{LecB}$ variants interacting with $\mathrm{Le}^{\mathrm{x}}$, an unusual conformation is observed for the $N$-acetyl group of all four $\mathrm{Le}^{\mathrm{x}}$ molecules of the asymmetric unit. In all cases, the $\mathrm{C} 2-\mathrm{N}$ bond adopts a non-canonical syn orientation of the $\alpha$ dihedral angle (Fig. 1D, Table 1). A detail of the electron density is displayed in Fig. S1. The $\beta$ torsion angles always remain in the classical trans conformation (Fig. 1D, Table 1). In all the binding sites, the unusual conformation of the $\mathrm{N}$-acetyl brings the bulky acetyl group away from the protein surface. The NH moiety points in the direction of the acidic group of Asp96, establishing a direct hydrogen bond in $\mathrm{LecB}_{\mathrm{PAO} 1}$, and a water bridged one in $\mathrm{LecB}_{\mathrm{PA} 14}$ (Table 1). The presence of $\mathrm{S} 97$ in the $\mathrm{LecB}_{\mathrm{PA} 14} / \mathrm{Le}^{\mathrm{x}}$ complex and accompanying hydration results therefore in slightly different position and hydrogen bond network from the $\mathrm{LecB}_{\mathrm{PAO} 1} / \mathrm{Le}^{\mathrm{x}}$ complex.

Table 1. Geometric characteristics of $N$-acetyl of $\mathrm{Le}^{\mathrm{x}}$ in the crystal structures of complexes with LecB ${ }_{\mathrm{PA} 14}$ and LecB $\mathrm{PAO}_{\mathrm{P}} \alpha$ and $\beta$ torsion angles $\left({ }^{\circ}\right)$ are defined as $\alpha(\mathrm{H} 2-\mathrm{C} 2-\mathrm{N}-\mathrm{H})$, $\beta\left(\mathrm{H}-\mathrm{N}-\mathrm{C} 1^{\prime}-\mathrm{O} 1^{\prime}\right)$ and $\mathrm{d} 1(\AA)$ is the distance between GlcNAc:N and D96:OD1.

\begin{tabular}{ccccccc}
\hline & \multicolumn{2}{c}{$\alpha$} & \multicolumn{2}{c}{$\beta$} & \multicolumn{2}{c}{$\mathrm{d} 1$} \\
\cline { 2 - 7 } chain & LecB $_{\text {PA14 }}$ & LecB $_{\text {PAO1 }}$ & LecB $_{\text {PA14 }}$ & LecB $_{\text {PAO1 }}$ & LecB BA14 & LecB BAO1 \\
\hline A & -6.8 & -8.3 & 172.4 & 177.1 & 3.7 & 3.0 \\
B & -9.0 & -11.7 & 174.1 & 169.5 & 3.6 & 3.1 \\
C & -6.9 & -13.3 & 171.7 & 161.3 & 3.5 & 3.1 \\
D & -1.8 & 12.4 & 169.1 & 149.7 & 3.5 & 3.4 \\
\hline
\end{tabular}




\section{Analysis of GIcNAc $N$-acetyl group in the Protein Data Bank}

In order to evaluate the frequency of the rare non-canonical orientation of $\mathrm{N}$-acetyl, the GlcNAc residues were analyse in the Protein Data Bank (PDB). Such a study was previously performed for glycoproteins and protein/glycan complexes [21], but with no quality check on the electron density. The search (as of March 17, 2019) yielded altogether 381 conformers, with 338 in the canonical $\alpha$-anti/ $\beta$-trans conformation i.e. $88.7 \%$ of occurrences with dihedrals ranging from $139^{\circ}$ to $209^{\circ}\left(-151^{\circ}\right)$ for $\alpha$ and $140^{\circ}$ to $210^{\circ}\left(-150^{\circ}\right)$ for $\beta$ (Fig. 3 ).

The other observed structures can be assigned to two non-canonical conformations, syn/trans or anti/cis, and one outlier syn/cis. These minor populations were further analysed by direct examination of the electron density maps using Coot [22] and the structure which were not supported by the electron density were discarded. For syn/trans torsion combination, 16 cases were confirmed including 8 from this work, i.e. $4 \%$ occurrence with $\alpha$ ranging from $-10^{\circ}$ to $11^{\circ}$ and $\beta$ from $172^{\circ}$ to $185^{\circ}\left(-175^{\circ}\right.$ ) (Fig. 3). For anti/cis, only six case were retained, i.e. $1.6 \%$ occurrence with $\alpha$ ranging from $165^{\circ}$ to $188^{\circ}\left(-172^{\circ}\right)$ and $\beta$ from $-21^{\circ}$ to $1^{\circ}$ (Fig. 3 ). The syn/cis outlier was not supported by the electron density and as such it was discarded.

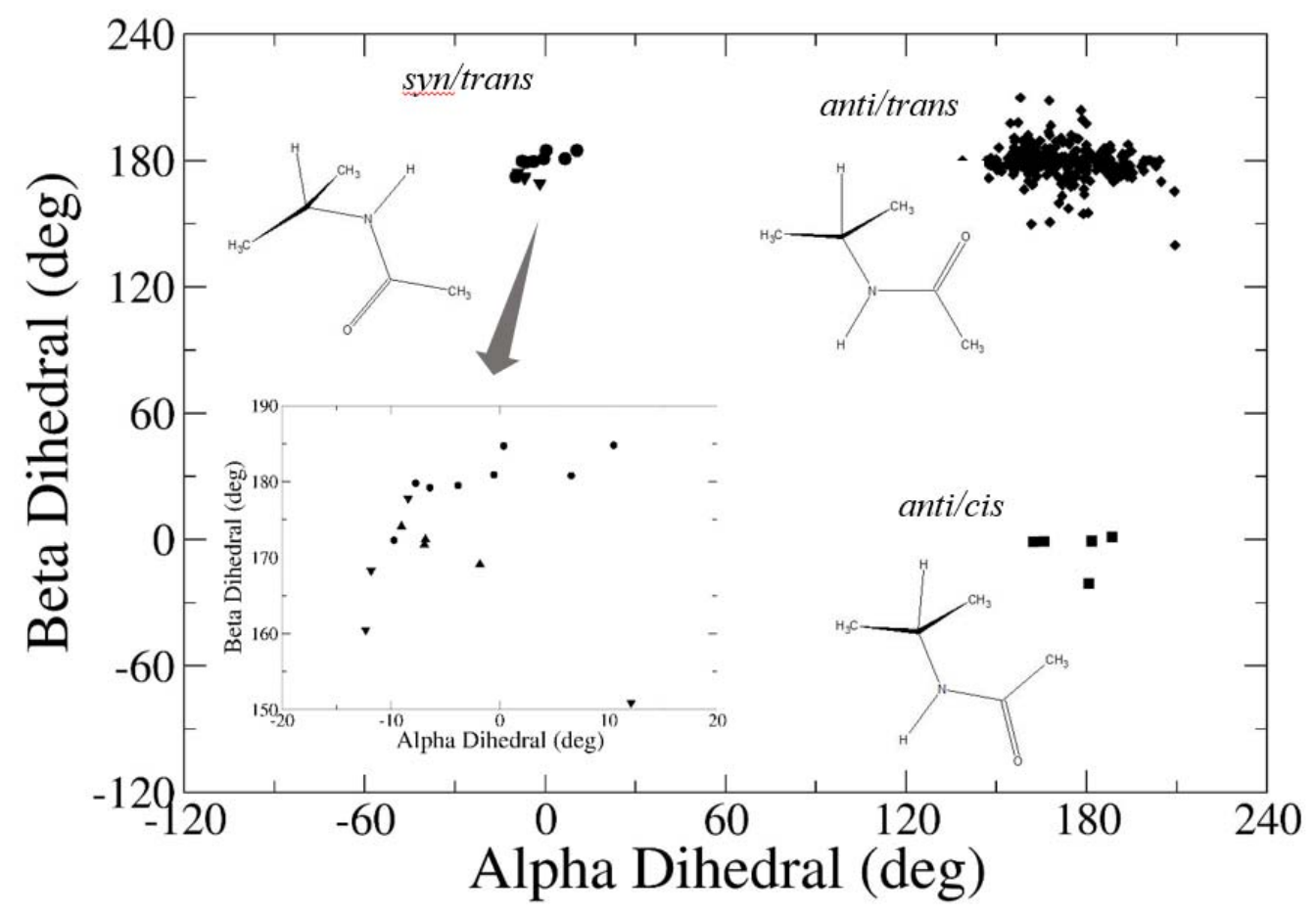

Figure 3. Analysis of $N$-acetyl $\alpha$ and $\beta$ dihedral angles in $\beta$-D-GlcNAc in protein/carbohydrate complexes in the PDB (each point represents one monosaccharide in a PDB structure). The relative orientation of the functional groups in the minor populations is 
shown schematically. Inset: Zoom in the $\alpha$-syn/ $\beta$-trans region (crystal structures determined in this work; LecB $\mathrm{PA} 14_{4} / \mathrm{Le}^{\mathrm{x}}$, triangle up; $\mathrm{LecB}_{\mathrm{PAO} 1} / \mathrm{Le}^{\mathrm{x}}$, triangle down).

Analysis of the PDB complemented with examination of the electron density maps confirmed eight cases plus another eight observed here crystallographically (i.e. $4 \%$ of the torsions studied) of the $N$-acetyl $\alpha$ dihedral in syn conformation

\section{Conformational behaviour of the $N$-Acetyl group in $\mathrm{Le}^{\mathrm{x}}$}

The $\mathrm{Le}^{\mathrm{x}}$ trisaccharide conformational behaviour was previously studied by us [14] by molecular dynamics using the GLYCAM06-j force field [23] in explicit water (TIP3P model) [24]. Several independent trajectories of 1 to $10 \mu$ s were produced, starting from the $\mathrm{Le}^{\mathrm{x}}$ solution shape with the $N$-acetyl group in the canonical conformation. The behaviour of the $\mathrm{N}$-acetyl group was analysed for the present work and no variations were observed for the $\mathrm{N}$ acetyl group in these trajectories (data not shown).

In the present work, a $1.4 \mu$ s MD simulation of $\mathrm{Le}^{\mathrm{x}}$ tetrasaccharide in TIP3P water was started from the $\alpha$-syn conformation from the $\mathrm{LecB}_{\mathrm{PA} 14} / \mathrm{Le}^{\mathrm{x}}$ complex crystal structure (chain A, see above). We observed a rapid conversion within $50 \mathrm{~ns}$ to $\alpha$-anti and its persistence until the end of the simulation (Fig. 4A). The $\beta$ dihedral stayed in all the cases in trans in range from $147^{\circ}$ to $221^{\circ}\left(-139^{\circ}\right)$ (Fig. 4A). Therefore, the $\mathrm{MD}$ of the $\mathrm{Le}^{\mathrm{x}}$ in aqueous solution confirms the stability of the major anti/trans conformation observed in most of the protein/carbohydrate complexes in the PDB. However, the determination of the rotational barrier is necessary in order to obtain trajectories with relevant occupancy of the different states.
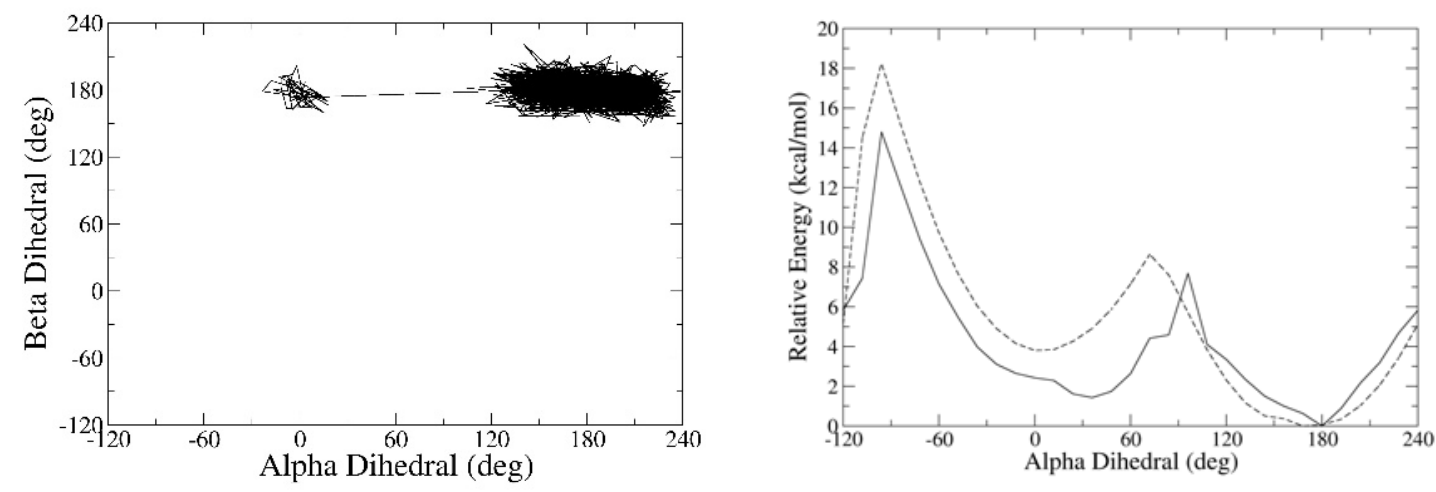

Figure 4. Conformational stability of the $N$-acetyl $\alpha$ dihedral of $\mathrm{Le}^{\mathrm{x}}$ tetrasaccharide. A. MD/explicit solvent. Syn/trans $\left(0 / 180^{\circ}\right)$ changed to anti/trans $\left(180 / 180^{\circ}\right)$ after $50 \mathrm{~ns}$ and stayed until $1.4 \mu \mathrm{s}$. B. The relaxed rotational scan of the $N$-acetyl $\alpha$ dihedral in $\mathrm{Le}^{\mathrm{x}}$ using quantum mechanics (solid line) and molecular mechanics (dashed line) calculations in implicit solvent (for details, see text). 
The relaxed rotational scans of the $\alpha$ dihedral in $\mathrm{Le}^{\mathrm{x}}$ were calculated at two levels (Fig. 4B). First, for reference, we used quantum mechanics (QM; density functional theory with empirical dispersion (DFT-D3)[25] with B-LYP functional and double- $\zeta$ basis set (DZVP) [26] coupled with implicit solvent COSMO model [27] in Turbomole [28].Second, for comparison with the MD simulations, we used molecular mechanics (MM; GLYCAM06j)[23] coupled with generalised Born (GB) implicit solvent using the igb5 parametrisation [29] in AMBER18 [30]. Overall, both levels of theory give similar shapes of the curves. The locations and relative stabilities of the minima in $\mathrm{Le}^{\mathrm{x}}$ mirror the populations found in the PDB (c.f. Fig. 3) and MD (Fig. 4A). The global minimum is at $\alpha$-anti $\left(180^{\circ}\right)$ and the local minimum at $\alpha-\operatorname{syn}\left(0^{\circ}\right.$; relative energy to the minimum of the method is $3.8 \mathrm{kcal} / \mathrm{mol}$ for MM and $36^{\circ}$ and $1.4 \mathrm{kcal} / \mathrm{mol}$ for QM). Barriers to rotation of the $N$-acetyl $\alpha$ dihedral from syn to anti are asymmetric. The high barriers at $-96^{\circ}$ of $14.5 \mathrm{kcal} / \mathrm{mol}$ (i.e. $18.3-3.8 \mathrm{kcal} / \mathrm{mol}$ at $0^{\circ}$ ) in $\mathrm{MM}$ and $13.4 \mathrm{kcal} / \mathrm{mol}$ (i.e. $14.8-1.4 \mathrm{kcal} / \mathrm{mol}$ at $36^{\circ}$ ) in $\mathrm{QM}$ are brought about by the repulsions between GlcNAc:O1' carbonyl oxygen and GlcNAc:O3, which also induces unfavourable amide bond non-planarity (Fig S2A). The smaller barrier of $4.8 \mathrm{kcal} / \mathrm{mol}$ (i.e. $8.6-3.8 \mathrm{kcal} / \mathrm{mol}$ at $0^{\circ}$ ) at $72^{\circ}$ for $\mathrm{MM}$ and $6.3 \mathrm{kcal} / \mathrm{mol}$ (i.e. $7.7-1.4 \mathrm{kcal} / \mathrm{mol}$ at $36^{\circ}$ ) at $96^{\circ}$ for QM is reduced due to stabilising H-bonds between the GlcNAc $N$-acetyl and the adjacent monosaccharide units (GlcNAc:N2-H ${ }^{\cdots}$ Fuc:O2 and reducing Gal:O2-H20 ${ }^{\cdots}$ GlcNAc:O1'; Fig. S2B). For a backward rotation (i.e. anti-to-syn), the barrier is higher -8.6 or 7.7 $\mathrm{kcal} / \mathrm{mol}$ in $\mathrm{MM}$ or $\mathrm{QM}$, respectively, which suggests why we could not observe any such transition in $1.4 \mu \mathrm{s} \mathrm{MD}$.

The $\beta$ dihedral angle stayed trans in all the $\alpha$ rotational profile optimisations which is not surprising given the high rotational barriers of $19-20 \mathrm{kcal} / \mathrm{mol}$ calculated from NMR experiments [21]. We have optimised $\mathrm{Le}^{\mathrm{x}}$ tetrasaccharide with anti/trans and anti/cis conformations at the QM level and the latter was higher in energy by $2.6 \mathrm{kcal} / \mathrm{mol}$. The reasons behind this finding are the intrinsic low stability of the $\beta$ cis isomer as found for GlcNAc by NMR [21] and also the lack of stabilising intramolecular H-bonds.

\section{Molecular dynamics of $\mathrm{LecB} / \mathrm{Le}^{\mathrm{x}}$ complex}

To gain insight into the stabilisation of the rare $\alpha$ dihedral in the crystallographic $\mathrm{LecB}_{\mathrm{PA} 14} / \mathrm{Le}^{\mathrm{x}}$ complex, we performed $600 \mathrm{~ns}$ MD simulations using the standard force fields for the protein (ff14SB) [31], carbohydrates (GLYCAM06-j) [23], water (TIP3P) [24] and $\mathrm{Ca}^{2+}$ ions [32] (Setup 1, Table 2). In line with previous observations [19, 20], the distance between the two calcium ions increased from the crystallographic value of $3.74 \AA$ (Fig. $5 \mathrm{~A}$, yellow) to $4.74 \AA$ (average distance after $150 \mathrm{~ns}$, Fig. 5A, black) due to a too high repulsion. 
A more advanced set of calcium parameters employing the $C_{4}$ charge-induced dipole term [33] was thereafter used (Setup 2) but with a similar outcome - the average distance after 200 ns was $4.73 \AA$ (Fig. 5A, red). To model the known charge transfer effects [4, 34] implicitly, we used calcium parametrization including effective polarization [35] (Setup 3). The agreement with the experiment was excellent with average values of $3.80 \pm 0.15 \AA$ (Fig. 5A, green). Refining further the latter approach, the $\mathrm{Ca}^{2+}$-coordinating carboxylates may be scaled for yet improved description [36]. This setup (Setup 4) gave a fair agreement with the crystallographic distance $\left(4.07 \pm 0.14 \AA\right.$; Fig. 5A, blue). Nonbonded parameters for $\mathrm{Ca}^{2+}, \mathrm{K}^{+}$ and $\mathrm{Cl}^{-}$ions in different MD setups are gathered in Table S3. Partial charges on the $\mathrm{Ca}^{2+}$ coordinating carboxylates in different MD setups are gathered in Table S4.

Table 2. Summary of the tested MD protocols with different parameterization of calcium ions: 12-6-IOD [32], 12-6-4 [33] or ECCR [35].

\begin{tabular}{cccc}
\hline Setup & $\mathrm{Ca}^{2+}$ & Protein & Water \\
\hline 1 & $12-6-$ IOD & ff14SB & TIP3P \\
2 & $12-6-4$ & ff14SB & TIP3P \\
3 & ECCR & ff14SB & TIP3P \\
4 & ECCR & ff14SB/scaled carboxylates & TIP3P \\
5 & ECCR & ff14SB & SPC/E \\
6 & ECCR & ff14SB & OPC3 \\
7 & ECCR & ff14SB & TIP4P \\
\hline
\end{tabular}

Further, we analysed the behaviour of the $\alpha$ torsion throughout the MD using Setups 3 and 4, since they maintain the best the architecture of the binding site (as opposed to Setups 1 and 2, c.f. Fig. 5A). Moreover, we tested three other water models in combination with the scaled $\mathrm{Ca}^{2+}$ parameters [35] because we knew from the crystal structure that water molecule networks bridge LecB with Le ${ }^{\mathrm{x}}$. Besides the classical TIP3P model (Setup 3), these additional water models are: another three-site rigid SPCE model [37] (Setup 5), a more recent threesite OPC3 model [38] (Setup 6) and TIP4P four-site model [39] (Setup 7).

In Fig. 5B (green) we see that MD with Setup 3 maintained the $N$-acetyl group in the $\alpha$-syn conformation only partially (for 85\%). After $170 \mathrm{~ns}$, it departed to $\alpha$-anti for $90 \mathrm{~ns}$ but thereafter it stayed at $\alpha$-syn until the end of the trajectory. In Setup 4 (Fig. 5B, blue), the $\alpha-$ syn conformation was maintained for $88 \%$ of the simulation time, i.e. until $530 \mathrm{~ns}$, then it changed to $\alpha$-anti and stayed until the end of the simulation. For the three water models in combination with the scaled-charge $\mathrm{Ca}^{2+}$ model, the $\alpha$-syn conformation was retained even for a larger proportion of time (99, 100 and 98\%, for Setup 5, 6, and 7, respectively). 


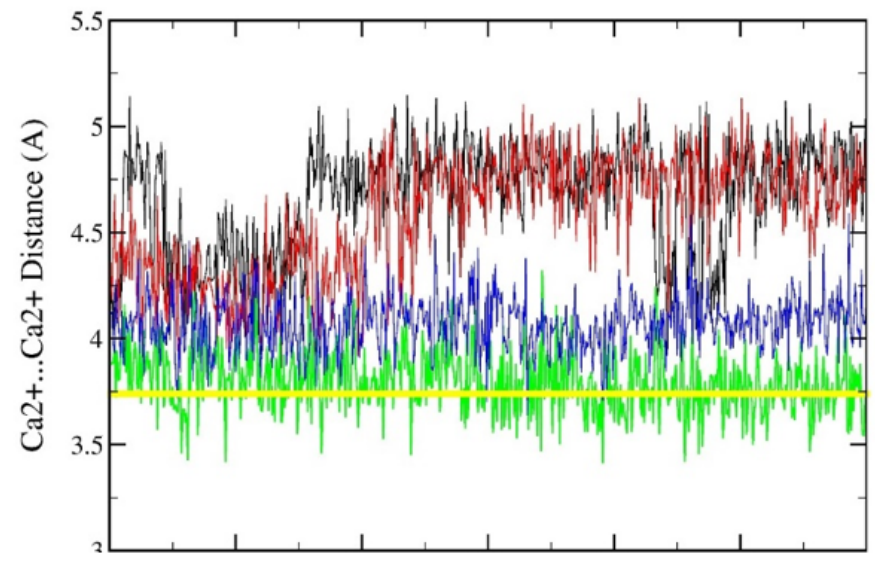

B

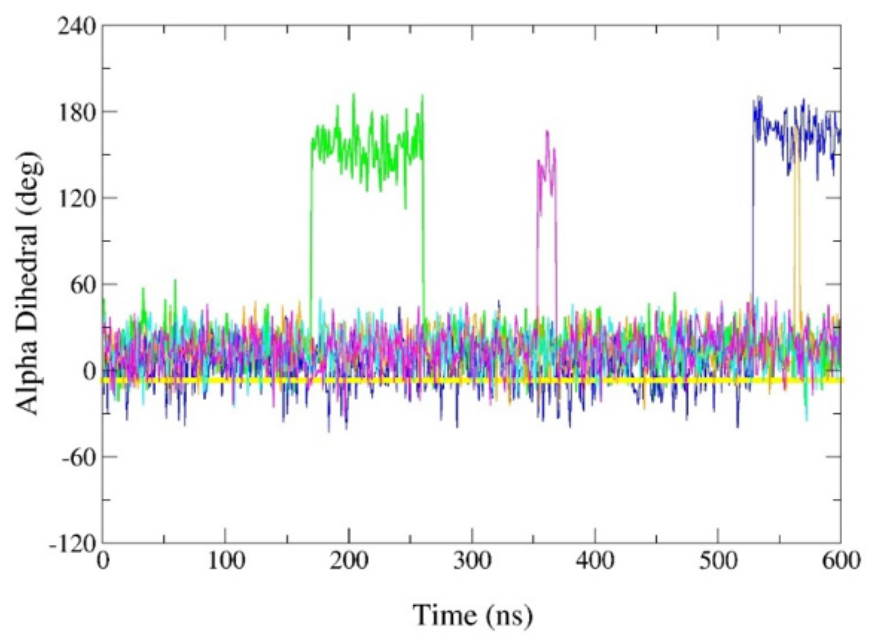

C

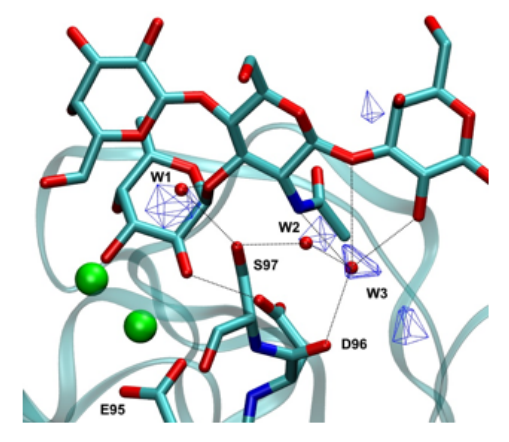

D

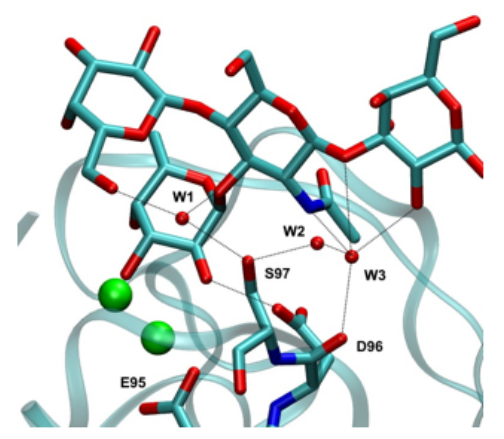

Figure 5. Analyses of $600 \mathrm{~ns} \mathrm{MD}$ simulations of $\mathrm{LecB}_{\mathrm{PA} 14} / \mathrm{Le}^{\mathrm{x}}$ complex and comparison with the crystal structure. A. The $\mathrm{Ca}^{2+\ldots} \mathrm{Ca}^{2+}$ distance and B. the $N$-acetyl $\alpha$ torsion. Curve colour coding: MD simulations with different setups: black - Setup 1; red - Setup 2; green - Setup 3; blue - Setup 4, orange - Setup 5, cyan - Setup 6, magenta - Setup 7. The LecB ${ }_{\mathrm{PA} 14} / \mathrm{Le}^{\mathrm{x}}$ crystal structure (chain A) determined here - yellow. Zoom at the interaction between LecB (ribbon) and $\mathrm{Le}^{\mathrm{x}}$ (sticks) from $\mathbf{C}$. a representative snapshot from MD using Setup 4 and D. the LecB $\mathrm{PA} 14_{4} / \mathrm{Le}^{\mathrm{x}}$ crystal structure. Atom colour coding: cyan - carbon, red - oxygen, blue nitrogen. Hydrogens are omitted from the MD snapshot for clarity. Dotted lines indicate $\mathrm{H}-$ bonding. Blue meshes show densities of water oxygen atoms contoured at 0.06 isosurface value. The densities are $0.112,0.082$ and 0.069 for $\mathrm{W} 1, \mathrm{~W} 2$, and $\mathrm{W} 3$, respectively. 
A more detailed insight into the reliability of the computational description of the $\mathrm{LecB}_{\mathrm{PA} 14} / \mathrm{Le}^{\mathrm{x}}$ complex is obtained by monitoring two distances. For the interactions of the $\mathrm{N}$ acetyl, it is the D96:OD $\cdots$ GlcNAc:N distance (d1) which in the $\mathrm{LecB}_{\mathrm{PA} 14} / \mathrm{Le}^{\mathrm{x}}$ crystal structure is $3.7 \AA$ in chain A (c.f. Table 1), i.e. it slightly exceeds the criterion for H-bond formation. For the role of Ser97 in organizing the W1-W3 water network, we monitor the Ser97:OG $\cdots$ D96:OD distance (d2) which in the crystal structure shows no signs of hydrogen bonding (4.6 $\AA$ ). In all the MD protocols tested, d1 varies between 2.8 and $4.5 \AA$ but averages to $3.2 \AA$ (Fig. S3), i.e. forming a direct hydrogen bond, not observed in the $\mathrm{LecB}_{\mathrm{PA} 14} / \mathrm{Le}^{\mathrm{x}}$ crystal structure. We note however that i) a direct H-bond has also been observed in the closely related $\mathrm{LecB}_{\mathrm{PA} 14} / \mathrm{Le}^{\mathrm{x}}$ crystal structure and ii) that even the direct H-bonding did not prevent transient formation of bifurcated H-bond toward W3 site, which in turn mediated the bridge to D96:O (c.f. Fig. 2). The d2 distance evolved during MD towards a non-native strong H-bond (distance of $2.6 \AA$ ), except Setups 4 and 7. In Setup 7, a strong Ser97:OG ${ }^{\cdots}$ D96:OD H-bond $(2.6 \AA)$ was formed for the starting $400 \mathrm{~ns}$ and then was lost. But Ser97 then bulged out to the solvent to a non-native position. In Setup 4, Ser97:OG is not attracted so much by D96:OD, due to the reduced charges on the carboxylates and thus does not make the non-native $\mathrm{H}$-bond. This results in an excellent reproduction of the details of the carbohydrate binding site including the location of the $\mathrm{Ca}^{2+}$ ions and water networks in Setup 4 as compared to the crystal structure (Fig. 5C, D). Taken together, the charge-scaling procedure on the calcium ions and their coordinating carboxylates (Setup 4, ECCR2) provides the most balanced description of the dynamics in the $\mathrm{LecB} / \mathrm{Le}^{\mathrm{x}}$ complex.

\section{Discussion and Conclusions}

The properties of isolated GlcNAc have been previously studied by a combination of experimental (NMR) and computational (QM, MD) techniques, which yielded a molecularlevel understanding of the structures, dynamics and energetics of several conformational families. Regarding the $N$-acetyl group in GlcNAc, the global minimum of the $\alpha$ dihedral is predicted to be anti $\left(161^{\circ}\right.$ and $180^{\circ}$ for the $\alpha$ and $\beta$ anomers, respectively) [40]. The shift in the minimum between the anomers is caused by intramolecular hydrogen bonding. In the $\beta$ anomer, a minor population (13\%) of $\alpha$-syn conformation was predicted from a $5 \mathrm{~ns}$ MD simulation [40]. We note, however, that this percentage is dependent both, on the force-field type and version and on the length of simulation, all variables that have been improved since [41]. The $\beta$ amide bond dihedral was found by NMR to be predominantly in trans (98\%) with a high barrier of $19-20 \mathrm{kcal} / \mathrm{mol}$ to conversion to $\beta$ cis [21].

In oligosaccharides containing GlcNAc, especially those where it serves as a branching point, the location and relative stabilities of the minima of the $\alpha$ dihedral along with the transition barriers may change due to the molecular surroundings. Indeed, we found that a QM 
rotational profile of $\mathrm{Le}^{\mathrm{x}}$ tetrasaccharide was governed by repulsive oxygen/oxygen contacts on one hand and stabilising $\mathrm{O}-\mathrm{H} \cdots \mathrm{O}$ hydrogen bonds, thus shaping the potential energy curve. The GLYCAM06 carbohydrate force field performed qualitatively well in locating the minima but overestimated their energy difference. This translated into the instability of the $\alpha-$ syn dihedral in a $1.4 \mu$ s-long MD of $\mathrm{Le}^{\mathrm{x}}$ in explicit solvent, in agreement with expected lower energy barriers in explicit solvent. Additional relaxation of the system may be due to the pyranose ring chair pucker transitions from the most stable ${ }^{4} C_{l}$ chair, which however occurs rarely $[14,42,43]$.

A PDB-wide search showed that GlcNAc-containing oligosaccharides bound to proteins exhibited one major (anti/trans) and two minor (syn/trans and anti/cis) populations (c.f. Fig. 3 ), for which we inspected the experimental electron densities and found that only 16 syn/trans cases (including 8 determined in this work, i.e. $4 \%$ of the torsions studied) could be confirmed unequivocally. In contrast, for the $\beta$ dihedral, in the six cases with good fit in the electron densities we could not confirm the minor anti/cis population because the $s p^{2}$ substituents could both be fitted upon $180^{\circ}$ rotation. Our two crystal structures of $\mathrm{LecB} / \mathrm{Le}^{\mathrm{x}}$ complexes showed that the geometry and characteristics of the LecB binding site were sufficient to stabilise the rare $\alpha$-syn conformation of $N$-acetyl of GlcNAc. The free energy cost associated with this rearrangement was partly compensated for by acquired hydrogen bonding (direct or water bridged) between the NH group of the $\mathrm{N}$-acetyl and D96, as could be observed in the crystal structures.

The LecB carbohydrate-binding site presents a particularly challenging system for force-field calculations due to the charge delocalisation induced by the two closely positioned calcium ions [4]. Previous modelling studies of LecB complexed with monosaccharides indicated problems arising from modelling this special site using the standard $2+$ charge model of calcium ion - the $\mathrm{Ca}^{2+} \ldots \mathrm{Ca}^{2+}$ distance rose by up to $20 \%$ with respect to the crystallographic one. $[19,20]$. Furthermore, the present structures of LecB/Le ${ }^{\mathrm{x}}$ complexes determined add another layer of complexity due to the rare $\alpha$ torsion of GlcNAc, whose force-field parametrisation needs to be validated against QM data. Lastly, the presence of water networks mediating protein/carbohydrate interactions requires testing of several water models. A new paradigm in modelling $\mathrm{Ca}^{2+}$ ions via effective polarisation had been proposed [44] and developed for inorganic salts, proteins and lipids [35, 36, 45]. The strategy is to scale calcium ion (and counterion) charges by 0.75 , reparametrize Lennard-Jones parameters and optionally scale the charged groups in biomolecules. Herein, both these approaches applied to the $\mathrm{LecB} / \mathrm{Le}^{\mathrm{x}}$ complex yielded results superior to standard treatment of calcium ions with $2+$ partial charge. Upon testing three additional water models (SPCE, OPC3 and TIP4P) coupled with scaling $\mathrm{Ca}^{2+}$ and counterions only, we obtained a better agreement with the crystallographic finding. Finally, we utilised scaled-charge $\mathrm{Ca}^{2+}$ ions and counterions 
which were coupled with TIP4P water model. This setup gave an excellent agreement with the experimental $\mathrm{Ca}^{2+} \ldots \mathrm{Ca}^{2+}$ distance and an excellent preservation of the crystallographic $\alpha-$ syn dihedral of $\mathrm{Le}^{\mathrm{x}}$ although with appearance of new hydrogen bonds. The second promising setup (Setup 4) utilised scaled-charge $\mathrm{Ca}^{2+}$ ions, counterions and $\mathrm{Ca}^{2+}$-coordinating carboxylates and the system was immersed in TIP3P water model. Such an approach gave poorer but still acceptable $\mathrm{Ca}^{2+} \ldots \mathrm{Ca}^{2+}$ distance but less preservation of the rare unstable $\alpha-s y n$ dihedral of $\mathrm{Le}^{\mathrm{x}}$. On the contrary, due to the scaling of the binding site carboxylates, occurrence of non-native H-bonding is avoided.

In conclusion, to the best of our knowledge, this is the first report on comprehensive testing of force-field parametrisations on protein with two closely positioned calcium ions in the binding site complexed with a carbohydrate in a rare conformation. By identifying one promising setup, we pave the way for further development, exploration and fine-tuning of parameters to obtain a general protocol for reliable description of such difficult systems where quantum phenomena play important roles. Even though further improvements of force fields are needed for a balanced handling of charge delocalization, the present study allowed for a better understanding of protein/carbohydrate interactions, which can in turn be applied for design of glycomimetics of therapeutical interest.

\section{Methods}

X-ray crystallography

Molecular cloning, expression and purification of $\mathrm{LecB}_{\mathrm{PA} 14}$ and $\mathrm{LecB}_{\mathrm{PAO} 1}$ were performed as previously described $[4,6]$. For crystallization, either $\operatorname{LecB}_{\mathrm{PA} 14}$ or $\mathrm{LecB}_{\mathrm{PAO} 1}$ was dissolved in water $\left(10 \mathrm{mg} \mathrm{mL}^{-1}\right)$ and incubated with $800 \mu \mathrm{g} \cdot \mathrm{mL}^{-1}$ of $\mathrm{Le}^{\mathrm{x}}$ tetrasaccharide (Elicityl) supplemented with $2 \mathrm{mM} \mathrm{CaCl} 2$ for 30 minutes prior to crystallization experiment. Crystallization was performed by the hanging drop vapour diffusion method using $1 \mu \mathrm{L}$ of the protein solution with $\mathrm{Le}^{\mathrm{x}}$ and $1 \mu \mathrm{L}$ of the reservoir solution containing $28 \%$-30\% PEG8K, $2 \mathrm{mM} \mathrm{CaCl}_{2}, 0.2 \mathrm{M}$ ammonium sulphate, and $0.1 \mathrm{M}$ Tris- $\mathrm{HCl} \mathrm{pH} 8.5$ at $19{ }^{\circ} \mathrm{C}$ in a 24 well plate. Crystals were cryo-protected with well solution supplemented with $10-25 \%(\mathrm{v} / \mathrm{v})$ glycerol and flash-cooled in liquid nitrogen. Data were collected at ESRF-BM30A (Grenoble, France) for LecB $\mathrm{PA} 14_{4}$ or at SOLEIL-PROXIMA1 (Saint Aubin, France) for LecB $\mathrm{P}_{\mathrm{PAO} \text {, using }}$ an ADSC Q315 CCD detector and a Pilatus 6M hybrid photon counting detector (Dectris), respectively. The recorded data were indexed, integrated and scaled using XDS [46] and merged using AIMLESS [47]. The structures were solved by molecular replacement with PHASER [48] using the PDB entry 5A6Q (for LecB PA14 $_{-L^{x}}$ ) or $1 \mathrm{~W} 8 \mathrm{H}$ (for LecB $\mathrm{PAO}-\mathrm{Le}^{\mathrm{x}}$ ) as 
a searching template. The model was finalised by further iterations of manual rebuilding in COOT [22] and restrained refinement in REFMAC5 [49]. Ligand libraries were created using Ligand Builder in Coot and built by Acedrg in CCP4 suite [50]. The geometries of the final models were validated with MOLPROBITY [51], wwPDB validation service (https://validate-rcsb-1.wwpdb.org/) and PDB-redo (https://pdb-redo.eu/) before submission to the Protein Data Bank. All structural figures were prepared using CCP4MG [52]. Data processing and refinement statistics are provided in Table S1 in the Supplementary Information.

\section{Protein Data Bank (PDB) Search}

The values of the $\mathrm{N}$-acetyl $\alpha$ and $\beta$ dihedrals of $\beta$-D-GlcNAc pyranose residues in protein/carbohydrate complexes were measured in the PDB database using the GlyTorsion tool [53]. To increase the confidence of the structural parameters, we set the X-ray resolution cutoff to $1.5 \AA$. NMR structures were filtered out for the lack of a quality validation criterion. To be able to observe the effect of the neighbouring carbohydrate moieties, we set the minimal oligosaccharide chain length to trisaccharide. The GlyTorsion tool measures the $\omega 2 \mathrm{a}$ $(\mathrm{C} 1-\mathrm{C} 2-\mathrm{N}-\mathrm{C} 1$ ') and $\omega 2 \mathrm{~b}(\mathrm{C} 2-\mathrm{N}-\mathrm{C} 1$ '-O1') dihedrals ("x-ray definition", i.e. using heavy atoms only, c.f. Fig. 1D). These values were transformed into a more readily understandable "NMR definition" [7] (using hydrogens added via LEaP program of AMBER18 [30]: $\alpha$ dihedral (H2-C2-N-H) and $\beta$ dihedral (H-N-C-O) by adding the approximate value of $60^{\circ}$ (deviations of several degrees from the exact values, data not shown) and the exact value of $180^{\circ}$, respectively. This approach avoids the need of adding hydrogens to the structures in a PDB-wide analysis.

\section{Modelling}

All the modelling was done on $\mathrm{A}$ chain (or $\mathrm{A} / \mathrm{B}$ chain dimer) of $\mathrm{LecB}_{\mathrm{PA} 14} / \mathrm{Le}^{\mathrm{x}}$ structure because of the high quality of the electron density maps of the whole $\mathrm{Le}^{\mathrm{x}}$ tetrasaccharide. Crystallographic water molecules were retained except those with partial occupancy which may induce clashes. Hydrogens were added using the LEaP program of AMBER18 [30]. As suggested by the previous crystal structure and semiempirical quantum chemical calculations [4], the carboxylates in the active site were left unprotonated. The molecular mechanics (MM) force fields used were: ff14SB [31] for the protein and GLYCAM-06j [23] for the carbohydrate. Several $\mathrm{Ca}^{2+}$ parametrisations were used: standard nonbonded +2 charge [32] optimized for ion-oxygen distance (IOD) (Setup 1); more advanced one +2 charge and employing the $C_{4}$ charge-induced dipole term (Setup 2) [33], a setup with charge on calcium and counterions scaled by 0.75 (Setup 3) [35] and lastly a setup inspired by [36] where Setup 3 was augmented with scaling $\mathrm{Ca}^{2+}$-coordinating carboxylates by 0.75 (Setup 4; partial charges are in Table S4). Several water models were used in conjunction with the scaled 
calcium and counterion setup: TIP3P [24] (Setup 3), SPCE [37](Setup 5), OPC3 [38](Setup 6) and TIP4P [39] (Setup 7). Hydrogens were optimized using the generalized Born (GB) implicit solvent model [29] ( $\varepsilon_{\mathrm{r}}$ of 78.5) by 5000 cycles of LBFGS optimization with zeroed dihedral barriers, followed by another 5000 LBFGS cycles in full force field.

Quantum Chemical Calculations

$N$-Acetyl Rotational Scan. The $\alpha$ dihedral in the $\mathrm{Le}^{\mathrm{x}}$ tetrasaccharide was rotated to $0^{\circ}$ using Cuby4 [54]. Thereafter it was rotated stepwise by $12^{\circ}$ in either direction up to a dihedral of $180 / 180^{\circ}$ and optimised in each step to tight convergence criteria (maximum energy difference of $0.6 \mathrm{cal} / \mathrm{mol}$, maximum gradient of $0.12 \mathrm{kcal} / \mathrm{mol} / \AA^{2}$ ), keeping the $\alpha$ dihedral fixed. This was performed in Cuby4 [54] at MM/GB level (using GLYCAM06-j [23] for Le ${ }^{\mathrm{x}}$ and $i g b=5$ option for generalised Born implicit solvation) using AMBER18 [30] or QM/COSMO level (DFT-D3 using B-LYP functional/DZVP basis set [26]) with COSMO [27] implicit solvent using Turbomole 7.0 [28]. Energies of the relaxed structures relative to the minimum of the respective method were plotted.

QM Charge Calculations. The LecB $\mathrm{PA}_{\mathrm{PA} 14}$ chain $\mathrm{A} / \mathrm{B}$ dimer with two $\mathrm{Le}^{\mathrm{x}}$ tetrasaccharides, four calcium ions and the crystallographic water molecules had its hydrogen atoms optimized by use of MM/GB [29]. Thereafter, it was subjected to 600 cycles of further hydrogen optimization at the semiempirical QM (SQM) level using PM6-D3H4 [55] coupled with COSMO [27] using MOPAC2016 (http://OpenMOPAC.net). The resulting structure was subjected to another 600 cycles of hydrogen optimization at QM/SQM/COSMO level, QM being B-LYP/DZVP-D3 [26]. We used a subtractive mechanical embedding scheme with link hydrogen atom approach. The QM part consisted of residues within $4.8 \AA$ of the two calcium ions in the A chain, the $\mathrm{Le}^{\mathrm{x}}$ tetrasaccharide and one crystallographic water molecule (W1), altogether 262 atoms. The SQM part comprised the whole system. For charge calculations, Natural Bond Orbital (NBO) [56] method was used on the isolated QM part or isolated and its QM/COSMO optimized constituents

\section{Molecular Dynamics}

The $\mathrm{LecB}$ chain $\mathrm{A} / \mathrm{B}$ dimer with two $\mathrm{Le}^{\mathrm{x}}$ tetrasaccharides, four calcium ions and the crystallographic water molecules with MM/GB optimized hydrogens was immersed in an octahedral box of water molecules which extended at least $12 \AA$ from the solute. $\mathrm{K}^{+} / \mathrm{Cl}^{-}$ counterions (scaled in Setups 3 and 4; their Lennard-Jones parameters are shown in Table S3) were added to maintain neutrality and physiologic concentration of $0.15 \mathrm{M}$. Bonds involving hydrogen atoms were constrained using SHAKE [57]. Hydrogen mass repartitioning to 3Da [58] was applied, which allowed us to use a longer integration time step of 4 fs. Initial 
stepwise relaxation and subsequent MD was performed according to the published protocol [59].

\section{ASSOCIATED CONTENT}

The crystallographic methodology and analyses, QM optimised structures, MD analyses and MD parameters are presented in the Supporting Information.

\section{CONTRIBUTORS}

M. Lepsik, AI and AT designed the study. RS and SK performed the crystallography work under the guidance of AV. M. Lepsik performed all calculations with the help of M. Lelimousin and EP. M. Lepsik, SK and AI wrote the manuscript.

\section{ACKNOWLEDGEMENT}

Dr. Martin Lepsik has received funding for this project from the European Union's Horizon 2020 research and innovation programme under the Marie Sklodowska-Curie grant agreement No 795605. The authors acknowledge support by the ANR PIA Glyco@Alps (ANR-15-IDEX-02), Labex ARCANE and CBH-EUR-GS (ANR-17-EURE-0003). Further, we acknowledge funding from the Helmholtz Association (grant no. VH-NG-934). We are grateful to synchrotron SOLEIL (Saint Aubin, France) and ESRF (Grenoble, France) for access and technical support at beamline PROXIMA 1 and BM30A, respectively. Part of the computations presented in this paper were performed using the Froggy platform of the CIMENT infrastructure which is supported by the Rhône-Alpes region (GRANT CPER07_13 CIRA) and the Equip@Meso project (reference ANR-10-EQPX-29-01). The work has been performed under the Project HPC-EUROPA3 (INFRAIA-2016-1-730897), with the support of the EC Research Innovation Action under the H2020 Programme; in particular, the author gratefully acknowledges the computer resources and technical support provided by EPCC at the University of Edinburgh, Scotland. We thank Emilie Gillon for providing recombinantly produced LecB $\mathrm{BAO}_{\mathrm{PA}}$ for X-ray crystallographic experiments.

\section{REFERENCES}

[1] N. Gilboa-Garber, Pseudomonas aeruginosa lectins, Methods Enzymol., 83 (1982) 378-385.

[2] A. Imberty, M. Wimmerova, E.P. Mitchell, N. Gilboa-Garber, Structures of the lectins from Pseudomonas aeruginosa: Insights into molecular basis for host glycan recognition, Microb. Infect., 6 (2004) 222-229.

[3] E. Mitchell, C. Houles, D. Sudakevitz, M. Wimmerova, C. Gautier, S. Pérez, A.M. Wu, N. GilboaGarber, A. Imberty, Structural basis for oligosaccharide-mediated adhesion of Pseudomonas aeruginosa in the lungs of cystic fibrosis patients, Nat. Struct. Mol. Biol., 9 (2002) 918-921. 
[4] E.P. Mitchell, C. Sabin, L. Šnajdrová, M. Pokorná, S. Perret, C. Gautier, C. Hofr, N. GilboaGarber, J. Koča, M. Wimmerová, A. Imberty, High affinity fucose binding of Pseudomonas aeruginosa lectin PA-IIL: $1.0 \AA$ resolution crystal structure of the complex combined with thermodynamics and computational chemistry approaches, Proteins, 58 (2005) 735-748.

[5] J.E. Heggelund, A. Varrot, A. Imberty, U. Krengel, Histo-blood group antigens as mediators of infections, Curr. Opin. Struct. Biol., 44 (2017) 190-200.

[6] R. Sommer, S. Wagner, A. Varrot, C. Nycholat, A. Khaledi, S. Häussler, J. Paulson, A. Imberty, A. Titz, The virulence factor LecB varies in clinical isolates: consequences for ligand binding and drug discovery, Chem. Sci., 7 (2016) 4990-5001

[7] X. Hu, I. Carmichael, A.S. Serianni, N-acetyl side-chains in saccharides: NMR J-coupling equations sensitive to $\mathrm{CH}-\mathrm{NH}$ and $\mathrm{NH}-\mathrm{CO}$ bond conformations in 2-acetamido-2-deoxyaldohexopyranosyl rings, J. Org. Chem., 75 (2010) 4899-4910.

[8] S. Perret, C. Sabin, C. Dumon, M. Pokorná, C. Gautier, O. Galanina, S. Ilia, N. Bovin, M. Nicaise, M. Desmadril, N. Gilboa-Garber, M. Wimmerova, E.P. Mitchell, A. Imberty, Structural basis for the interaction between human milk oligosaccharides and the bacterial lectin PA-IIL of Pseudomonas aeruginosa, Biochem. J, 389 (2005) 325-332.

[9] A.M. Boukerb, A. Decor, S. Ribun, R. Tabaroni, A. Rousset, L. Commin, S. Buff, A. DoleansJordheim, S. Vidal, A. Varrot, A. Imberty, B. Cournoyer, Genomic rearrangements and functional diversification of lecA and lecB lectin coding regions impacting the efficacy of glycomimetics directed against Pseudomonas aeruginosa, Frontiers Microb., 7 (2016) 811.

[10] A. Imberty, A. Varrot, Microbial recognition of human cell surface glycoconjugates, Curr. Opin. Struct. Biol., 18 (2008) 567-576.

[11] R.U. Lemieux, K. Bock, L.T.J. Delbaere, S. Koto, V.S.R. Rao, The conformations of oligosaccharides related to the ABH and Lewis human blood group determinants, Can. J. Chem., 58 (1980) 631-653.

[12] S. Pérez, N. Mouhous-Riou, N.E. Nifant'ev, Y.E. Tsvetkov, B. Bachet, A. Imberty, Crystal and molecular structure of a histo-blood group antigen involved in cell adhesion: the Lewis $\mathrm{x}$ trisaccharide, Glycobiology, 6 (1996) 537-542.

[13] M. Zierke, M. Smiesko, S. Rabbani, T. Aeschbacher, B. Cutting, F.H. Allain, M. Schubert, B. Ernst, Stabilization of branched oligosaccharides: Lewis(x) benefits from a nonconventional CH...O hydrogen bond, J. Am. Chem. Soc., 135 (2013) 13464-13472.

[14] J. Topin, M. Lelimousin, J. Arnaud, A. Audfray, S. Pérez, A. Varrot, A. Imberty, The hidden conformation of Lewis $\mathrm{x}$, a human histo-blood group antigen, is a determinant for recognition by pathogen lectins, ACS Chem. Biol., 11 (2016) 2011-2020.

[15] S. Cecioni, A. Imberty, S. Vidal, Glycomimetics versus multivalent glycoconjugates for the design of high affinity lectin ligands, Chem. Rev., 115 (2015) 525-561.

[16] A. Imberty, Y.M. Chabre, R. Roy, Glycomimetics and glycodendrimers as high affinity microbial antiadhesins, Chem. Eur. J., 14 (2008) 7490-7499.

[17] D. Hauck, I. Joachim, B. Frommeyer, A. Varrot, B. Philipp, H.M. Möller, A. Imberty, T.E. Exner, A. Titz, Discovery of two classes of potent glycomimetic inhibitors of Pseudomonas aeruginosa LecB with distinct binding modes, ACS Chem. Biol., 8 (2013) 1775-1784.

[18] R. Sommer, S. Wagner, K. Rox, A. Varrot, D. Hauck, E.-C. Wamhoff, J. Schreiber, T. Ryckmans, T. Brunner, C. Rademacher, R.W. Hartmann, M. Brönstrup, A. Imberty, A. Titz, First glycomimetic, orally bioavailable LecB inhibitors block biofilm formation of Pseudomonas aeruginosa, J. Am. Chem. Soc., 140 (2018) 2537-2545. 
[19] N.K. Mishra, P. Kulhánek, L. Šnajdrová, M. Petřek, A. Imberty, J. Koča, Molecular dynamics study of Pseudomonas aeruginosa lectin-II complexed with monosaccharides, Proteins, 72 (2008) 382-392.

[20] R. Sommer, T.E. Exner, A. Titz, A biophysical study with carbohydrate derivatives explains the molecular basis of monosaccharide selectivity of the Pseudomonas aeruginosa lectin LecB, PLoS One, 9 (2014) e112822.

[21] X. Hu, W. Zhang, I. Carmichael, A.S. Serianni, Amide cis-trans isomerization in aqueous solutions of methyl N-formyl-D-glucosaminides and methyl N-acetyl-D-glucosaminides: chemical equilibria and exchange kinetics, J. Am. Chem. Soc., 132 (2010) 4641-4652.

[22] P. Emsley, B. Lohkamp, W. Scott, K. Cowtan, Features and development of Coot, Acta Crystallogr. D. Biol. Crystallogr., 66 (2010) 486-501.

[23] K.N. Kirschner, A.B. Yongye, S.M. Tschampel, J. Gonzalez-Outeirino, C.R. Daniels, B.L. Foley, R.J. Woods, GLYCAM06: a generalizable biomolecular force field. Carbohydrates, J. Comput. Chem., 29 (2008) 622-655.

[24] W.L. Jorgensen, J. Chandrasekhar, J. Madura, M.L. Klein, Comparison of simple potential functions for simulating liquid water, J. Chem. Phys., 79 (1983) 926-935.

[25] S. Grimme, J. Antony, S. Ehrlich, H. Krieg, A consistent and accurate ab initio parametrization of density functional dispersion correction (DFT-D) for the 94 elements H-Pu, J. Chem. Phys., 132 (2010) 154104.

[26] J. Hostas, J. Rezac, Accurate DFT-D3 calculations in a small basis set, J. Chem. Theor. Comput., 13 (2017) 3575-3585.

[27] A. Klamt, G. Schüümann., COSMO: A new approach to dielectric screening in solvents with explicit expressions for the dcreening energy and its gradient., J. Chem. Soc. PerkinTrans. 2, (1993) 799-805.

[28] R. Ahlrichs, M. Bar, M. Haser, H. Horn, C. Kolmel, Electronic structure calculations on workstation computers: The program system Turbomole, Chem. Phys. Lett., 162 (1989) 165-169.

[29] A. Onufriev, D. Bashford, D.A. Case, Exploring protein native states and large-scale conformational changes with a modified generalized born model, Proteins, 55 (2004) 383-394.

[30] W.D. Cornell, P. Cieplak, C.I. Bayly, I.R. Gould, K.M.J. Merz, D.M. Ferguson, D.C. Spellmeyer, T. Fox, J.W. Caldwell, P.A. Kollman, A second generation force field for the simulation of proteins, nucleic acids, and organic molecules, J. Am. Chem. Soc., 117 (1995) 5179-5197.

[31] J.A. Maier, C. Martinez, K. Kasavajhala, L. Wickstrom, K.E. Hauser, C. Simmerling, ff14SB: Improving the accuracy of protein side chain and backbone parameters from ff99SB, J. Chem. Theor. Comput., 11 (2015) 3696-3713.

[32] P. Li, B.P. Roberts, D.K. Chakravorty, K.M. Merz, Jr., Rational design of Particle Mesh Ewald compatible Lennard-Jones parameters for +2 metal cations in explicit solvent, J. Chem. Theor. Comput., 9 (2013) 2733-2748.

[33] P. Li, K.M. Merz, Jr., Taking into account the Ion-induced dipole interaction in the nonbonded model of ions, J. Chem. Theor. Comput., 10 (2014) 289-297.

[34] M. Lepsik, M.J. Field, Binding of calcium and other metal ions to the EF-hand loops of calmodulin studied by quantum chemical calculations and molecular dynamics simulations, J Phys Chem B, 111 (2007) 10012-10022.

[35] M. Kohagen, M. Lepšík, P. Jungwirth, Calcium binding to calmodulin by molecular dynamics with effective polarization, J. Phys. Chem. Lett., 5 (2014) 3964-3969. 
[36] T. Martinek, E. Duboue-Dijon, S. Timr, P.E. Mason, K. Baxova, H.E. Fischer, B. Schmidt, E. Pluharova, P. Jungwirth, Calcium ions in aqueous solutions: Accurate force field description aided by ab initio molecular dynamics and neutron scattering, J. Chem. Phys., 148 (2018).

[37] H.J.C. Berendsen, J.R. Grigera, T.P. Straatsma, The missing term in effective pair potentials, J. Phys. Chem., 91 (1987) 6269-6271.

[38] S. Izadi, A.V. Onufriev, Accuracy limit of rigid 3-point water models, J. Chem. Phys., 145 (2016) 074501.

[39] W.L. Jorgensen, J.D. Madura, Temperature and size dependence for Monte Carlo simulations of TIP4P water, Mol. Phys., 56 (1985) 1381-1392.

[40] M. Mobli, A. Almond, N-Acetylated amino sugars: the dependence of NMR 3J(HNH2)couplings on conformation, dynamics and solvent, Org. Biomol. Chem., 5 (2007) 2243-2251.

[41] R.J. Woods, Predicting the structures of glycans, glycoproteins, and their complexes, Chem. Rev., 118 8005-8024.

[42] I. Alibay, K.K. Burusco, N.J. Bruce, R.A. Bryce, Identification of rare Lewis oligosaccharide conformers in aqueous solution using enhanced sampling molecular dynamics, J. Phys. Chem. B, 122 (2018) 2462-2474.

[43] B.M. Sattelle, A. Almond, Is N-acetyl-D-glucosamine a rigid 4C1 chair?, Glycobiology, 21 (2011) 1651-1662.

[44] I. Leontyev, A. Stuchebrukhov, Accounting for electronic polarization in non-polarizable force fields, Phys. Chem. Chem. Phys., 13 (2011) 2613-2626.

[45] J. Melcr, H. Martinez-Seara, R. Nencini, J. Kolafa, P. Jungwirth, O.H.S. Ollila, Accurate binding of sodium and calcium to a POPC bilayer by effective inclusion of electronic polarization, J. Phys. Chem. B, 122 (2018) 4546-4557.

[46] W. Kabsch, XDS, Acta Crystallogr., Sect D: Biol. Crystallogr., 66 (2010) 125-132.

[47] P.R. Evans, An introduction to data reduction: Space-group determination, scaling and intensity statistics, Acta Crystallogr., Sect D: Biol. Crystallogr., 67 (2011) 282-292.

[48] A.J. McCoy, Solving structures of protein complexes by molecular replacement with Phaser, Acta Crystallogr., Sect D: Biol. Crystallogr., 63 (2006) 32-41.

[49] G.N. Murshudov, P. Skubák, A.A. Lebedev, N.S. Pannu, R.A. Steiner, R.A. Nicholls, M.D. Winn, F. Long, A.A. Vagin, REFMAC5 for the refinement of macromolecular crystal structures, Acta Crystallogr., Sect D: Biol. Crystallogr., 67 (2011) 355-367.

[50] M.D. Winn, C.C. Ballard, K.D. Cowtan, E.J. Dodson, P. Emsley, P.R. Evans, R.M. Keegan, E.B. Krissinel, A.G.W. Leslie, A. McCoy, S.J. McNicholas, G.N. Murshudov, N.S. Pannu, E.A. Potterton, H.R. Powell, R.J. Read, A. Vagin, K.S. Wilson, Overview of the CCP4 suite and current developments, Acta Crystallogr., Sect D: Biol. Crystallogr., 67 (2011) 235-242.

[51] V.B. Chen, W.B. Arendall, J.J. Headd, D.A. Keedy, R.M. Immormino, G.J. Kapral, L.W. Murray, J.S. Richardson, D.C. Richardson, MolProbity: All-atom structure validation for macromolecular crystallography, Acta Crystallogr., Sect D: Biol. Crystallogr., 66 (2010) 12-21.

[52] S. McNicholas, E. Potterton, K.S. Wilson, M.E.M. Noble, Presenting your structures: The CCP4mg molecular-graphics software, Acta Crystallogr., Sect D: Biol. Crystallogr., 67 (2011) 386-394.

[53] T. Lutteke, M. Frank, C.W. von der Lieth, Carbohydrate Structure Suite (CSS): analysis of carbohydrate 3D structures derived from the PDB, Nucleic Acids Res., 33 (2005) D242-246. 
[54] J. Rezac, Cuby: An integrative framework for computational chemistry, J. Comput. Chem., 37 (2016) 1230-1237.

[55] J. Rezac, P. Hobza, Advanced corrections of hydrogen bonding and dispersion for semiempirical quantum mechanical methods, J. Chem. Theor. Comput., 8 (2012) 141-151.

[56] A.E. Reed, L.A. Curtiss, F. Weinhold, Intermolecular interactions from a natural bond orbital, donor-acceptor viewpoint, Chem. Rev., 88 (1988) 899-926.

[57] J.-P. Ryckaert, G. Ciccotti, H.J.C. Berendsen, Numerical integration of the cartesian equations of motion of a system with constraints: Molecular dynamics of n-alkanes, J. Comput. Phys., 23 (1977) 327-341.

[58] C.W. Hopkins, S. Le Grand, R.C. Walker, A.E. Roitberg, Long-time-step molecular dynamics through hydrogen mass repartitioning, J. Chem. Theor. Comput., 11 (2015) 1864-1874.

[59] P. Srb, M. Svoboda, L. Benda, M. Lepsik, J. Tarabek, V. Sicha, B. Gruner, K. Grantz-Saskova, J. Brynda, P. Rezacova, J. Konvalinka, V. Veverka, Capturing a dynamically interacting inhibitor by paramagnetic NMR spectroscopy, Phys. Chem. Chem. Phys., 21 (2019) 5661-5673. 